\title{
An ultraviolet diode laser system for laser cooling trapped ytterbium ions
}

\author{
J.A. Cox, M. Cetina, F.X. Kärtner \\ Research Laboratory of Electronics \\ Massachusetts Institute of Technology \\ Cambridge, United States
}

\author{
D. Kielpinski \\ Griffith University \\ Brisbane, Australia
}

\begin{abstract}
We describe the design and performance of an ultraviolet diode laser system for laser cooling trapped ytterbium ions without frequency doubling. The performance is similar to resonantly doubled laser systems, but with greater efficiency, reduced size, expense and fragility. The system achieved laser cooling of ytterbium ions in a linear Paul trap to less than $270 \mathrm{mK}$. No shorter wavelength laser has previously been used for laser cooling.
\end{abstract}

\section{INTRODUCTION}

Trapped ion laser cooling is the foundation for many promising technologies in optical frequency metrology and control. In particular, ytterbium ion laser cooling has been investigated for use in optical clocks. However, research and development of $\mathrm{Yb}^{+}$laser cooling, especially in space-based applications, has been retarded by the fragility, size, expense and inefficiency of the required frequency doubled infrared laser, such as a Ti-sapphire laser. However, recent advances in Gallium Nitride laser diode technology have enabled the development of ultraviolet (UV) lasers which can directly generate a short enough wavelength for ${ }^{172} \mathrm{Yb}^{+}$laser cooling. Thus, we describe a less expensive, smaller, simpler and more efficient technique for laser cooling $\mathrm{Yb}^{+}$using a UV diode to directly generate the required wavelength of 369.5 $\mathrm{nm}$. To the best of our knowledge, no shorter wavelength laser had previously been used for laser cooling. Furthermore, we demonstrate Doppler cooling of trapped $\mathrm{Yb}^{+}$ions to less than $270 \mathrm{mK}$ with this system. The output power and linewidth of the system is similar to resonantly doubled, single frequency Ti-sapphire lasers [1].

\section{TEMPERATURE TUNING}

Frequency control of the single mode GaN laser diode was achieved with a combination of holographic grating tuning, cavity length tuning and temperature cooling. A Nichia GaN laser diode (model NDHU110APAE2), with a room temperature, free running wavelength of approximately $375 \mathrm{~nm}$, was used in the system. GaN has a slow tuning response with temperature, which we measured as approximately $0.1 \mathrm{~nm}^{\circ} \mathrm{C}^{-1}$. Thus, it is necessary to lower the temperature of the diode significantly to achieve the required band gap energy of approximately $3.4 \mathrm{eV}$ and corresponding emission wavelength [2].

To achieve sufficient temperature cooling, two thermoelectric cooling (TEC) stages and a water cooled cold plate stage were used, as shown in Fig. 1. The precise temperature of the diode package was maintained with a proportional-integral-derivative (PID) temperature controller to $-23{ }^{\circ} \mathrm{C}$ with a 16 Watt TEC under the first stage. The second stage, consisting of two of the same TEC units, was cooled to approximately $-15{ }^{\circ} \mathrm{C}$ with a regulated power supply. Finally, the entire arrangement was sunk to a water chilled cold plate. In addition, insulation is required within the cold box, not necessarily to maintain low temperatures, but to prevent convection from inducing minute temperature fluctuations leading to frequency disturbances. Therefore, we used foam insulation to fill all unoccupied space. The entire laser system, excluding the cold plate, was encased in a

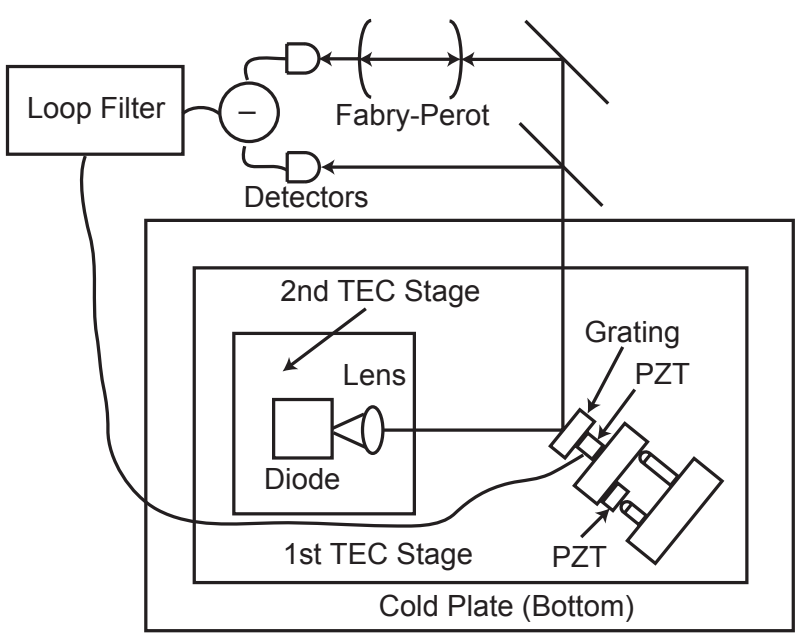

Figure 1. A diagram of the laser system, including the frequency control loop.

Supported by the Air Force Office of Scientific Research. 
sealed, polycarbonate box. To prevent condensation from damaging the diode and obscuring optical surfaces, the cold box was purged with Nitrogen prior to being cooled.

\section{LASER CAVITY}

The laser frequency was further tuned with holographic grating feedback by mounting the diode in a Littrow cavity arrangement as shown in Fig 1. The diode emission was collimated with an aspheric $\mathrm{f}=2.75 \mathrm{~mm}, 0.65$ numerical aperture lens. For optimum feedback, the lens was positioned slightly off of collimation. A 2400 line $\mathrm{mm}^{-1}$ diffraction grating was mounted on a mirror mount and adjusted so that the -1 order mode was back reflected to provide approximately $70 \%$ optical power feedback. Taking advantage of the frequency dependent angle of reflection of this mode (1), the angle of the grating was adjusted by hand as a course means of tuning the laser frequency to the edge of the gain curve.

$$
\theta=\sin ^{-1}\left(\frac{\lambda}{\Delta l}\right)
$$

Such adjustments were easily done in situ by flowing Nitrogen through the cold box, preventing contamination from the atmosphere. In addition, we observed that the original grease employed in the mirror mount became significantly more viscous at low temperatures, impeding adjustment. Fine adjustment of the grating angle was accomplished with a piezoelectric transducer (PZT) mounted within the mirror mount. This PZT was manually controlled after course adjustment of the mirror mount was achieved. Course adjustment was facilitated by directly monitoring the laser output with a wavemeter device so that a wavelength of approximately $369.5 \mathrm{~nm}$ was obtained.

\section{FREQUENCY CONTROL}

Frequency control of the laser system was accomplished with the sidelock technique, as shown in Fig 2. A PZT was mounted behind the grating so that the cavity length could be finely adjusted by translating the grating in a direction normal to the grating. A frequency reference was provided by a scanning Fabry-Perot cavity adjusted to the $\mathrm{Yb}^{+}$ resonance. A balanced photodetector and a PID loop filter, as shown in Fig. 2, was arranged to lock the laser frequency to the side of a Fabry-Perot resonance. The side of this resonance is where sensitivity to frequency fluctuations is greatest. This arrangement also allows the laser frequency to be scanned within a small range by varying the Fabry-Perot cavity length with the PZT in the Fabry-Perot. In addition, it was necessary to vary the diode current to achieve a lock at $369.5 \mathrm{~nm}$. Furthermore, this method allows for measurements of in loop laser noise at the photodetector.

It was necessary to antireflection coat one diode facet to obtain sufficient tuning resolution and reduce the occurance of mode hops of the diode cavity. Electron beam evaporation was performed while observing the threshold current of the diode. Upon reaching a threshold current just below the damage threshold of the device, the electron beam evaporation was terminated.

After antireflection coating, we measured a threshold current of $35.5 \mathrm{~mA}$ at $-23{ }^{\circ} \mathrm{C}$. We obtained an output power of $400 \mu \mathrm{W}$ with a diode current of $45 \mathrm{~mA}$. Since we observed a substantial rise in diode junction voltage for a given current as temperature was reduced, greater output power was inhibited by the maximum specified junction voltage of $6 \mathrm{~V}$. It was necessary to carefully monitor this voltage to prevent damage and achieve maximum possible output power.

The performance of the laser system is comparable to resonantly doubled Ti-sapphire lasers. By observing noise in the frequency control loop at the balanced photodetector, we found laser frequency stability exceeding $1 \mathrm{MHz}$ on a one second timescale - as shown by integrating over a detection band of $1 \mathrm{~Hz}$ to $1 \mathrm{MHz}$. Long term drift averaged $30 \mathrm{MHz}$ per minute. Moreover, from the laser cooling spectra we estimate frequency drifts of $\pm 0.5 \mathrm{MHz}$ per second from thermal fluctuations in the Fabry-Perot, which was made of Invar alloy. Also, we found that the diode experienced mode hops of the residual diode cavity at the rate of about once per hour.
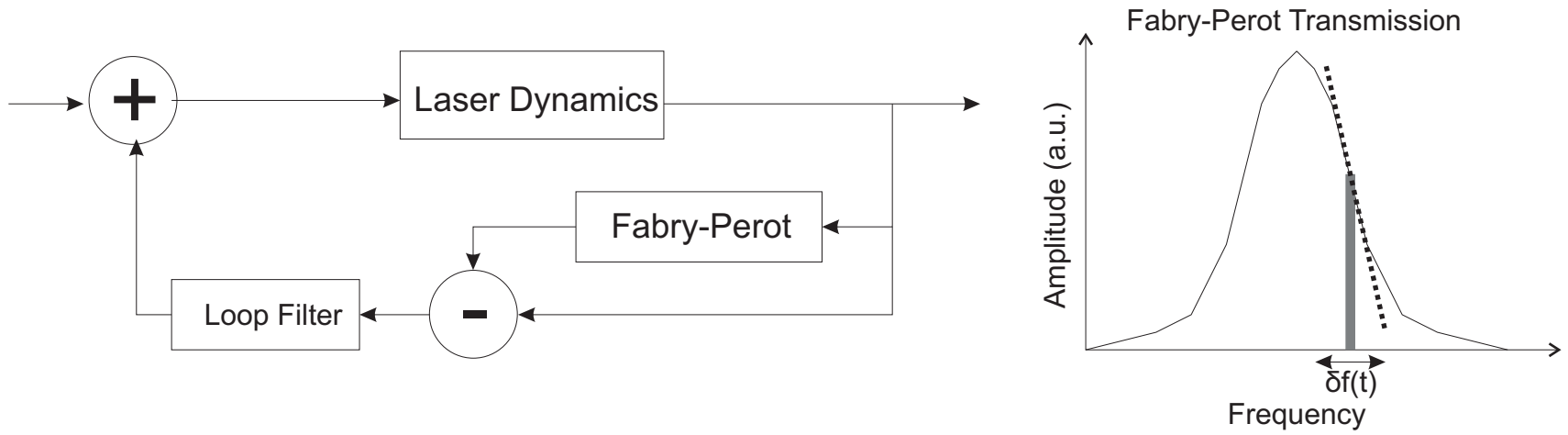

Figure 2. Shown on the left is a signal diagram of the sidelock technique. On the right is a plot of transmission verses frequency for the Fabry-Perot. The gray line represents the laser spectrum. The dotted line represents the slope of the filter profile at the laser frequency. 
temperatures below $270 \mathrm{mK}$. [1]

$$
\mathrm{T}=\frac{\mathrm{mc}^{2}}{8 \mathrm{k}_{\mathrm{B}} \ln 2} \frac{\Delta \mathrm{f}_{\mathrm{FWHM}}}{\mathrm{f}}
$$

\section{LASER COOLING}

Laser cooling of ${ }^{172} \mathrm{Yb}^{+}$was performed with the Doppler cooling technique in a linear Paul trap loaded with a mixture of approximately 100 ions in naturally occurring isotope abundances. The trap pseudopotential was provided by a 500 $\mathrm{V}, 1.9 \mathrm{MHz}$ signal applied to opposing trap terminals. The cooling cycle of ${ }^{172} \mathrm{Yb}^{+}$, as shown in Fig. 3, consists of the primary $369.5 \mathrm{~nm} \mathrm{~S} \mathrm{~S}_{1 / 2}-\mathrm{P}_{1 / 2}$ transition, which is interrupted by spontaneous decay to the metastable $\mathrm{D}_{3 / 2}$ state. An infrared diode "repump" laser, dithered around the $935 \mathrm{~nm}$ $\mathrm{D}_{3 / 2}-{ }^{3}[3 / 2]_{1 / 2}$ transition, pumps the ions into the ${ }^{3}[3 / 2]_{1 / 2}$ state, whereby it rapidly decays to the ground state, restoring the cooling cycle.

Analysis of the laser cooling performance was obtained by rapidly scanning the laser around the $\mathrm{Yb}^{+}$resonance and observing off axis ion fluorescence, as shown in Fig. 4, with a photomultiplier tube. The scan was performed by detuning the laser to the red of the resonance by several hundred Mhz and rapidly scanning past resonance. The resulting emission spectrum is a convolution of homogenous broadening from the natural $\mathrm{Yb}^{+}$linewidth and inhomogeneous Doppler broadening, known as a Voigt profile. Furthermore, it exhibits an arctangent profile owing to the sudden heating which occurs when the laser frequency reaches zero detuning, expanding the Doppler distribution greatly until few ions are near resonance. The data show a linewidth of 45 $\pm 3 \mathrm{MHz}$, as compared to the natural $\mathrm{Yb}^{+}$linewidth of 19.8 MHz. From the observed linewidth, we deduce, from (2), ion

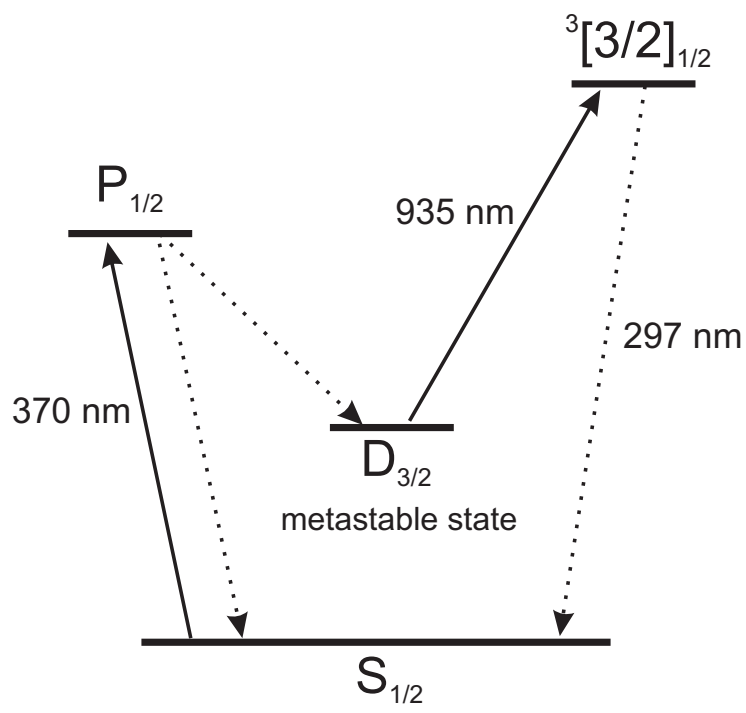

Figure 3. The $\mathrm{Yb}^{+}$laser cooling cycle is shown above. Solid lines represent induced transitions while dashed lines represent spontaneous transitions.

\section{CONCLUSION}

We have demonstrated laser cooling of $\mathrm{Yb}^{+}$with the direct generation of UV laser light from a laser diode. Performance of the laser is comparable to resonantly doubled Ti-sapphire lasers. No shorter wavelength laser has ever been used for laser cooling. Since we could not adjust the laser power with the detuning frequency (although such a capability can be implemented with the system in the future), the ion fluorescence spectrum is quite power broadened. Furthermore, cooling only the ${ }^{172} \mathrm{Yb}^{+}$isotope, instead of a mixture, would further improve cooling. Expanding the top TEC stage to contain both the grating mount and the laser diode could eliminate mode hops by reducing thermal fluctuations in the laser cavity. Future work may include replacing the Fabry-Perot frequency reference with a ytterbium vapor tube to eliminate the careful tuning and thermal fluctuations associated with the Fabry-Perot.

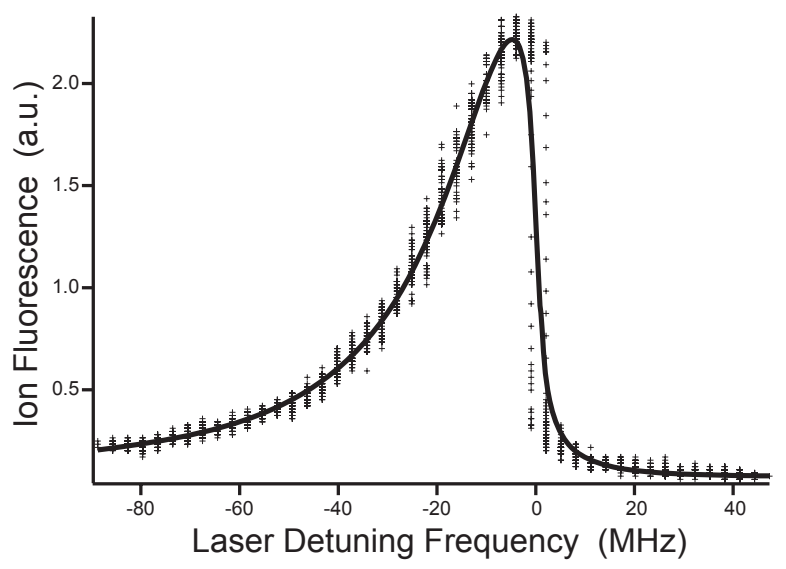

Figure 4. A plot of ion fluorescence verses laser detuning frequency from $\mathrm{Yb}^{+}$resonance at $369.5 \mathrm{~nm}$. Data points are shown with the best fit Voigt profile with arctangent softstep [1]. 


\section{ACKNOWLEDGMENTS}

We would like to acknowledge the US Air Force Office of Scientific Research for their support under contract F49620-03-1-0313 and the MIT Undergraduate Research Opportunities Program. In addition, we thank Peter O’Brian at the MIT Lincoln Laboratory for helping to antireflection coat the diodes. Finally, we also wish to thank those who provided helpful advice and insight.

\section{REFERENCES}

[1] D. Kielpinski, M. Cetina, J.A. Cox, and F.X. Kärtner. "Laser cooling of trapped ytterbium ions with an ultraviolet diode laser." Optics Letters. Vol. 31. No. 6

[2] S. Adachi. Physical Properties of III-V Semiconductor Compounds. John Wiley and Sons. New York, New York. 1992. 